\title{
Alteration in Expression of miR-32 and FBXW7 Tumor Suppressor in Plasma Samples of Patients with T-cell Acute Lymphoblastic Leukemia
}

This article was published in the following Dove Press journal: Cancer Management and Research

\author{
Sanaz Mansouri ${ }^{1} *$ \\ Behzad Khansarinejad ${ }^{2, *}$ \\ Ghasem Mosayebi ${ }^{2}$ \\ Aziz Eghbali ${ }^{3}$ \\ Mahdieh Mondanizadeh (D) ${ }^{1,4}$ \\ 'Department of Biotechnology and \\ Molecular Medicine, Arak University of \\ Medical Sciences, Arak, Iran; \\ ${ }^{2}$ Department of Microbiology and \\ Immunology, Arak University of Medical \\ Sciences, Arak, Iran; ${ }^{3}$ Department of \\ Pediatrics, School of Medicine, Arak \\ University of Medical Sciences, Arak, \\ Iran; ${ }^{4}$ Molecular and Medicine Research \\ Center, Arak University of Medical \\ Sciences, Arak, Iran \\ *These authors contributed equally to \\ this work
}

Background: T-cell acute lymphoblastic leukemia (T-ALL) is an aggressive and malignant neoplasm that arises from the hematopoietic T-cell precursors. Inactivation of $F B X W 7$ gene is frequently observed in T-cell acute lymphoblastic leukemia, suggesting a significant tumor-suppressive role for $F B X W 7$ in the pathobiology of this leukemia. Considering the role of microRNAs in cell proliferation and regulation of apoptosis, the aim of this study was to identify novel oncogenic microRNAs that suppress $F B X W 7$ in patients with T-ALL.

Patients and Methods: The expression levels of two bioinformatically predicted microRNAs - miR-32 and miR-107 were compared in patients with T-ALL and a control group. A total of 80 plasma samples were subjected to RNA extraction, and the microRNA expression profiles were assessed by the RT-qPCR. The expression level of miR-103 was used as the endogenous reference for normalization of quantitative data.

Results: The plasma levels of miR-32 and miR-107 in patients with T-ALL were significantly higher $(5.65, \mathrm{P}<0.001)$ and lower $(0.432, \mathrm{P}=0.002)$, respectively. On the other hand, the expression levels of $F B X W 7$ gene were significantly downregulated by -76.9 fold in T-ALL patients $(\mathrm{P}<0.001)$. The results of the ROC curve analysis indicated that overexpression of miR-32 might be used to distinguish T-ALL patients with reasonable sensitivity and specificity.

Conclusion: miR-32 is considered as a novel oncomir that targets $F B X W 7$ and might have a role in the etiology or progression of T-ALL. Furthermore, miR-32 can potentially serve as a non-invasive biomarker for detection of T-ALL.

Keywords: biomarker, $F B X W 7$, T-ALL, microRNA

\section{Introduction}

T-cell acute lymphoblastic leukemia (T-ALL) is an aggressive hematologic malignancy results from leukemic transformation of developing thymocytes, ${ }^{1,2}$ representing nearly $25 \%$ of adult and $10-15 \%$ of childhood ALL cases. ${ }^{2,3}$ Several cytogenetic abnormalities and genetic aberrations have been associated with the etiology of T-ALL. ${ }^{1,4}$ Some functions including self-renewal, proliferation and survival, and blocked differentiation of precursor $\mathrm{T}$ cells are affected by these genetic aberrations. ${ }^{1}$

FBXW7 is a tumor suppressor protein that leads to increased stability of NOTCH1 protein. FBXW7 expression increases subsequent p53 stimulation. $^{5}$ Inactivation of FBXW7 gene is frequently detected in T-ALL cases. ${ }^{6}$ More specifically, mutations of FBXW7 were found in about $9-30 \%$ of T-cell acute
Correspondence: Mahdieh Mondanizadeh Department of Biotechnology and Molecular Medicine, Arak University of Medical Sciences, Arak, Iran

$\mathrm{Tel} / \mathrm{Fax}+98-8634173526$

Email m_mondanizadeh@yahoo.com 
lymphocytic leukemia cases, indicating that FBXW7 plays an important tumor-suppressive role in the pathobiology of TALL. ${ }^{5-7}$ On the other hand, numerous studies have demonstrated the significant role of miRNAs in the regulation of a wide range of biological processes, including proliferation, differentiation, migration, apoptosis, metabolism and the stress response. miRNAs are a class of small non-coding RNA molecules, between 19 and 24 nucleotides in length. ${ }^{8}$ miRNAs act by degrading their RNA targets or by suppressing the translation of mRNAs. Additionally, miRNAs have been indicated to operate as strategic regulators in the pathogenesis of some diseases, especially cancers. In tumorigenic cells, miRNAs have been found to be seriously dysregulated. ${ }^{9,10}$ A few miRNAs can promote lymphoblastic leukemogenesis through inducing the expression of oncogenes and suppressing apoptosis, impressing the progression and prognosis of the disease. ${ }^{11}$ Several studies have shown that miRNAs are stable in plasma and serum and that circulating molecules display unique profiles for each tumor. The stability of miRNAs in circulation might be due to their protection by exosome and microvesicle, Argonaute2 (Ago2), and high-density lipoprotein. ${ }^{12,13}$ It was also shown that miRNA signatures can be applied either in the diagnosis the T-ALL or evaluation of the prognosis of patients. ${ }^{11,14,15}$

In the current study, bioinformatics tools were applied to predict the most relevant miRNAs in human plasma that interact with FBXW7 gene products. The expression levels of the predicted miRNAs as well as FBXW7 mRNA were compared in the plasma samples of patients with T-ALL and control group, using RT-qPCR. The role of the predicted miRNAs was also evaluated as potential biomarkers for the diagnosis of T-ALL.

\section{Materials and Methods}

\section{Patients and Samples}

Forty patients and 40 healthy individuals were obtained from Valiasr Hospital (Arak, Iran) enrolled in this study. All patients diagnosed as ALL by FAB criteria with an expert hemato oncologist and categorized in T-ALL by flow cytometry method. The peripheral blood was collected and plasma was separated, the following study designed on this part of sample. As illustrated in Table 1, healthy individuals' and Patients' samples were related to 20 females and 20 males, with a median age of 45 years (range, 29 to 71 years) for patients group and 39 (range,
Table I Baseline Characteristics of Patients and Control Groups

\begin{tabular}{|c|l|l|}
\hline Parameter & Patients & $\begin{array}{l}\text { Healthy } \\
\text { Controls }\end{array}$ \\
\cline { 2 - 3 } & Number (\%) & Number (\%) \\
\hline $\begin{array}{l}\text { Gender } \\
\text { Female }\end{array}$ & & \\
Male & $20(50)$ & $20(50)$ \\
\hline Age & $20(50)$ & $20(50)$ \\
Median & & \\
Range & 45 & 39 \\
\hline Peripheral blood & $29-71$ & $26-65$ \\
Mean WBC count $\times 10^{9} / \mathrm{L}$ & 47.2 & \\
Mean Hb concentration gr/L & 9.8 & 14.7 \\
Mean Plt count $\times 10^{9} / \mathrm{L}$ & 94 & $28 \mathrm{I}$ \\
\hline
\end{tabular}

26-65 years) for healthy individuals. In diagnosed of T-ALL patients, European Group for the Immunological Characterization of Leukemias (EGIL) suggested the flow cytometry marker to diagnosed (positive for CD34, CD 52, CD2 and, CD7 antigen and negative for myeloid antigen like CD33 and CD 117). ${ }^{16}$ All patients had not received cancer-related treatment including chemotherapy, radiotherapy, or surgery. All samples were stored at $-70^{\circ} \mathrm{C}$ prior to analysis. All patients and healthy volunteers provided written informed consent and the study was approved by the Ethics Committee of Arak University of Medical Sciences, in accordance with the declaration of Helsinki (ethics number IR.ARAKMU.REC.1396.253).

\section{miRNA Predictions}

The nucleotide sequence of FBXW7 gene, with official name of F-box and WD repeat domain containing 7, was retrieved from NCBI (http://www.ncbi.nlm.nih.gov/gene). The obtained sequence was evaluated in most generally accepted bioinformatics resources to examine the potential miRNAs that target FBXW7. These websites include DIANA (http:// diana.imis.athena-innovation.gr/DianaTools/index.php? $\mathrm{r}=$ microT_CDS/index), miRBase (http://www.mirbase.org/), PicTar (https://pictar.mdc-berlin.de/), miRanda (http://www. microrna.org/microrna/home.do), miRTB (https://bio.tools/ mirtarbase), TargetScan (http://www.targetscan.org/), miRDB (http://mirdb.org), and miRNApath (https://www. bioconductor.org/packages/release/bioc/html/miRNApath. $\mathrm{html}$ ). Since different algorithms are used in these softwares, we made a score table to select the most consensus candidate miRNAs for subsequent experimental analyses. Finally, two 
microRNAs with the highest probability were chosen to experimentally evaluate their role in targeting FBXW7.

\section{Primer Design}

The sequences of the miRNAs were acquired from miRBase database. Reverse transcription-specific stemloop primers and gene-specific primers were designed using the AlleleID7 and GeneRunner software. The expression of miR-103 and GAPDH was used for normalization of predicted miRNAs and FBXW7 gene expressions, respectively. ${ }^{17-19}$ The specificity of the designed primers was designed using the nucleotide BLAST on NCBI. The sequences of the primers are presented in Table 2 .

\section{miRNA Extraction and Reverse}

\section{Transcription}

MicroRNAs were extracted from plasma samples using RNX-Plus kit (SinaClon, Iran) according to the manufacturer's instructions and $1 \mu \mathrm{g}$ of RNA was reverse-transcribed using the mixture of M-MLV enzyme (Vivantis, Malaysia), 1x RT-enzyme buffer, $400 \mu \mathrm{M}$ dNTP, and $1 \mu \mathrm{M}$ of specific stem-loop RT primers and incubated at $75{ }^{\circ} \mathrm{C}$ for $5 \mathrm{~min}$. The mixture was incubated at $25^{\circ} \mathrm{C}$ for $15 \mathrm{~min}, 37^{\circ} \mathrm{C}$ for 15 $\min , 42^{\circ} \mathrm{C}$ for $45 \mathrm{~min}$, and $10 \mathrm{~min}$ at $75^{\circ} \mathrm{C}$, in a thermal cycler (Eppendorf, Germany). Finally, the obtained cDNAs were stored at $-20^{\circ} \mathrm{C}$ prior to RT-qPCR analyses.

\section{Quantitative Real-Time PCR}

All RT-qPCR analyses were performed in a Light Cycler 96 instrument (Roche, Germany) using the SYBR Green PremixExRaq II (Yekta Tajhiz Azma, Iran), $1.5 \mu \mathrm{L}$ cDNA, and $0.3 \mu \mathrm{M}$ of each forward and reverse primers, and $5.1 \mu \mathrm{L}$ RNase-free water to adjust the reaction volume to $15 \mu \mathrm{L}$. The temperature profile was $95^{\circ} \mathrm{C}$ for $3 \mathrm{~min}, 40$ cycles of $95{ }^{\circ} \mathrm{C}$ for $10 \mathrm{~s}, 55^{\circ} \mathrm{C}$ for $15 \mathrm{~s}$ and $72{ }^{\circ} \mathrm{C}$ for $20 \mathrm{~s}$ Melting curve analysis was performed after amplifications from to $60^{\circ} \mathrm{C}$ to $96^{\circ} \mathrm{C}$ with a ramp rate of $0.2^{\circ} \mathrm{C} /$ second and continuous fluorescence acquisition. The relative expression was calculated by the comparative $\mathrm{Cq}$ method using the relative expression software tool (REST). ${ }^{20}$

\section{Statistical Analyses}

Relative expressions were analyzed by the REST 2009 and the data in graphs are expressed as the mean \pm SE. Receiver operating characteristic (ROC) curve analysis was performed to evaluate the diagnostic value of the miRNA level. All statistical analyses were calculated using SPSS software (version 16; SSPS Inc., 184 Chicago). P-values of $<0.05$ were considered to indicate statistical significance.

\section{Results}

\section{Selection of the Predicted miRNAs}

The predictions of DIANA, miRBase, PicTar, miRanda, miRTB, TargetScan, miRDB, and miRNApath software's showed that miR-32, miR-27a and miR-107 are among the miRNAs that target FBXW7 transcript with the highest scores. Although different mathematical algorithms and scoring criteria used in different websites, the selected miRNAs were chosen based on the most repeated miRNA results (Table 3). Between these three miRNAs, miR-32 and miR-107 showed better complementarities with the target and were more consensus in targeting the

Table 2 Primers Used for Reverse-Transcription and RT-qPCR Assay of the Target miRNAs and FBXW7

\begin{tabular}{|c|c|}
\hline Target & Primer Sequences $\left(5^{\prime}-3^{\prime}\right)$ \\
\hline miR-32 & 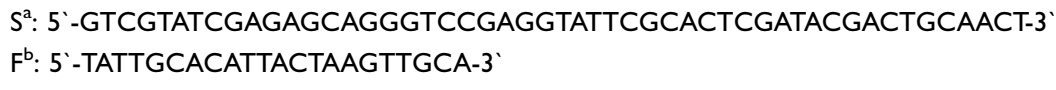 \\
\hline miR-I07 & $\begin{array}{l}\text { S: } 5 \text { '-GTCGTATCGAGAGCAGGGTCCGAGGTATTCGCACTCGATACGACAGCAGCA-3’ } \\
\text { F: } 5 \text { '-GCCCTGTACAATGCTGC-3’' }\end{array}$ \\
\hline miR-I03 & $\begin{array}{l}\text { S: } 5^{\prime} \text {-GTCGTATCGAGAGCAGGGTCCGAGGTATTCGCACTCGATACGACCAAGGCA-3’ } \\
\text { F: } 5 \text { '-GCTTCTTTACAGTGCTGCC-3' }\end{array}$ \\
\hline Common Reverse & 5'-AGAGCAGGGTCCGAGGT-3’ \\
\hline FBXW7 & $\begin{array}{l}\text { F: } 5^{\prime} \text {-AAACATTGCAAGGTCCCAAC-3' } \\
\text { R: } 5^{\prime} \text {-CTTTGTGTTTGAGGCTCTGATC-3' }\end{array}$ \\
\hline GAPDH & $\begin{array}{l}\text { F:5 '-GGAGTCCACTGGCGTCTTCAC-3`' } \\
\text { R:5 '-GAGGCATTGCTGATGATCTTGAGG-3` }\end{array}$ \\
\hline
\end{tabular}

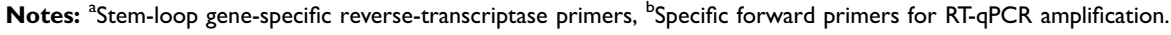


Table 3 The Result of miRNA Prediction for FBXW7

\begin{tabular}{|c|c|c|c|c|c|c|c|c|c|c|}
\hline Gene Name & miRNA & miRTB & TargetScan & miRDB & PicTar & miRanda & miRNApath & miRBase & DIANA & SUM \\
\hline FBXW7 & has-miR-32 & $I^{a}$ & I & I & I & I & I & I & I & 8 \\
\hline FBXW7 & has-miR-107 & I & 1 & 1 & 0 & I & I & 0 & 0 & 5 \\
\hline FBXW7 & has-miR-27a & $0^{\mathrm{b}}$ & I & 0 & I & I & 0 & I & 0 & 4 \\
\hline
\end{tabular}

Notes: ${ }^{a}$ Targeting of FBXW7 is confirmed by the software. ${ }^{\mathrm{b}}$ Targeting of FBXW7 is not confirmed by the software.

3'-UTR part of the FBXW7 transcript and were chosen for further experimental evaluation (Figure 1).

\section{Quantification of miRNA Plasma Levels}

As summarized in Figure 2, the RT-qPCR analyses showed that the expression levels of miR-32 were significantly higher in T-ALL patients than in healthy individuals (5.65, $\mathrm{P}<0.001)$, and the expression levels of miR-107 were significantly lower in T-ALL patients $(0.432, \mathrm{P}=$ 0.002). On the other hand, the expression levels of FBXW7 gene were significantly lower in T-ALL patients than in healthy individuals $(0.013, \mathrm{P}<0.001)$.

\section{Determination of Expression Level Cutoff}

Receiver operating characteristic (ROC) curve analyses were conducted to examine the cutoff values of miR-32 and miR-107 to differentiate healthy individuals from patients with T-ALL. The Youden's index ([sensitivity + specificity] -1) was used for the determination of appropriate cutoff levels of the ROC curves. Comparisons of the miRNA plasma levels between T-ALL patients and healthy individuals showed that at the cutoff level of 0.776, miR-107 had $82.6 \%$ sensitivity and $85.7 \%$ specificity with an area under curve (AUC) of 0.822 . For miR-32, at the cutoff level of 1.84 , the sensitivity was defined $95.2 \%$ and the specificity was $87 \%$. The AUC of the ROC for miR-32 was 0. 946 (Figure 3).

\section{Discussion}

Genetic and epigenetic alterations are recognized as one of the most important contributing factors for oncogenic transformation of immature T-cell progenitors. Currently, aberrant expression of miRNAs has been demonstrated to effect on the development and progression of different types of leukemia including T-ALL. ${ }^{15}$ miRNAs that target and repress the expression of tumor suppressor genes can play a critical role as oncogenes when aberrantly overexpressed in malignancy. ${ }^{21}$ The profiles of these miRNAs could differ based on the tumor type and, hence, might provide novel biomarkers for diagnosis of many cancers. ${ }^{22,23}$ It has been shown the expression levels of miR-181a, miR-128-3P and miR-142-3p were significantly up-regulated in T-ALL patients, ${ }^{1,4,24}$ whereas miR-204, miR-101 and miR-193b3 p were down-regulated. ${ }^{2,24,25}$

Some studies have indicated that FBXW7 dysregulates important signaling pathways including Notch1 and
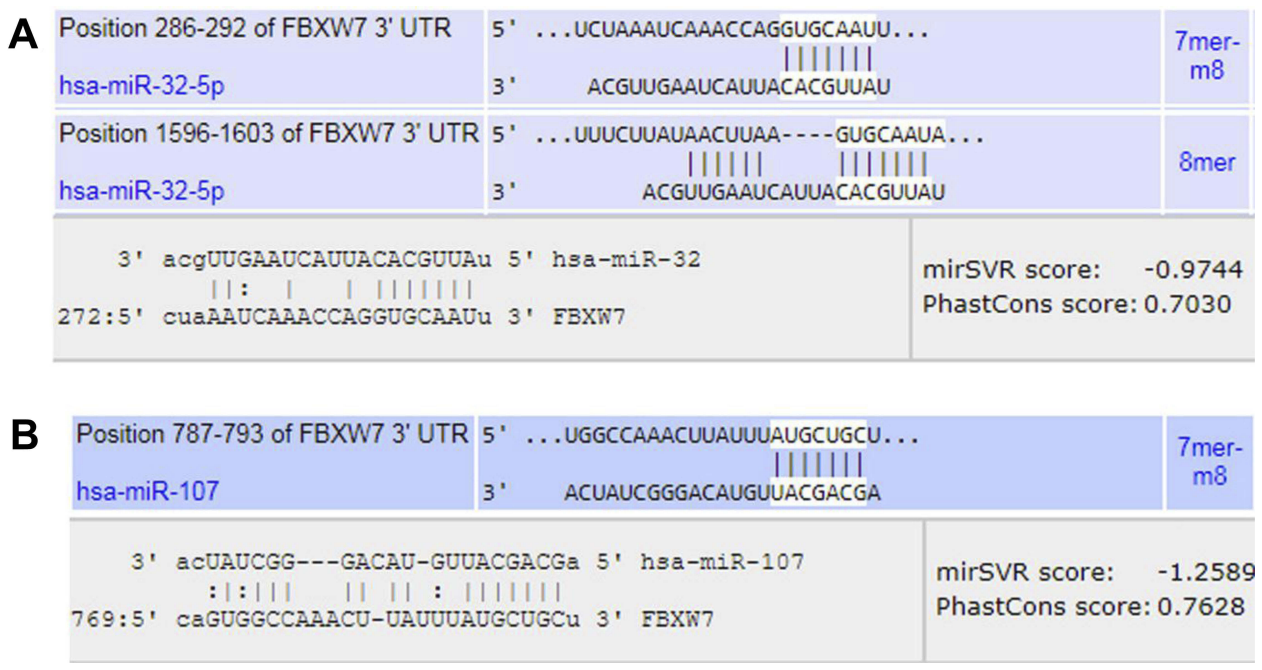

Figure I Schematic representation of the 3'-UTRs region of FBXW7 mRNA that is targeted by (A) miR-32 and (B) miR-I07 binding seed region. 

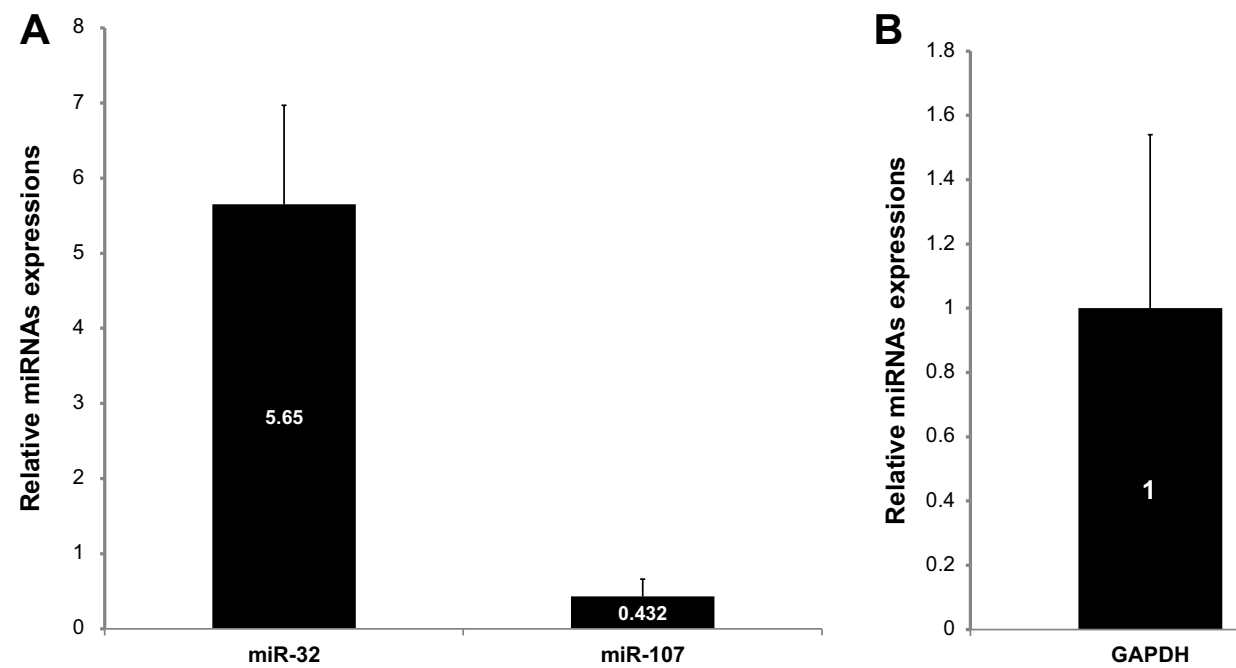

FBXW7

Figure 2 (A) Comparison of differential expression levels of miR-32 and miR-107 between patients with T-ALL and healthy individuals. (B) Relative expression of FBXW7 in T-ALL patients in comparison to healthy control group. Error bars indicate the standard error of the mean.

A

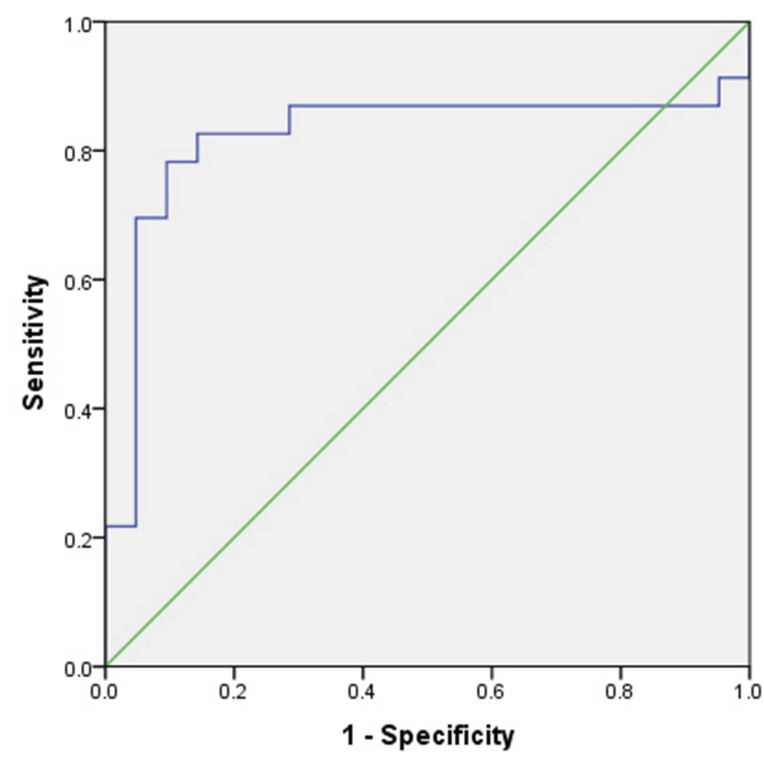

B

miR-32

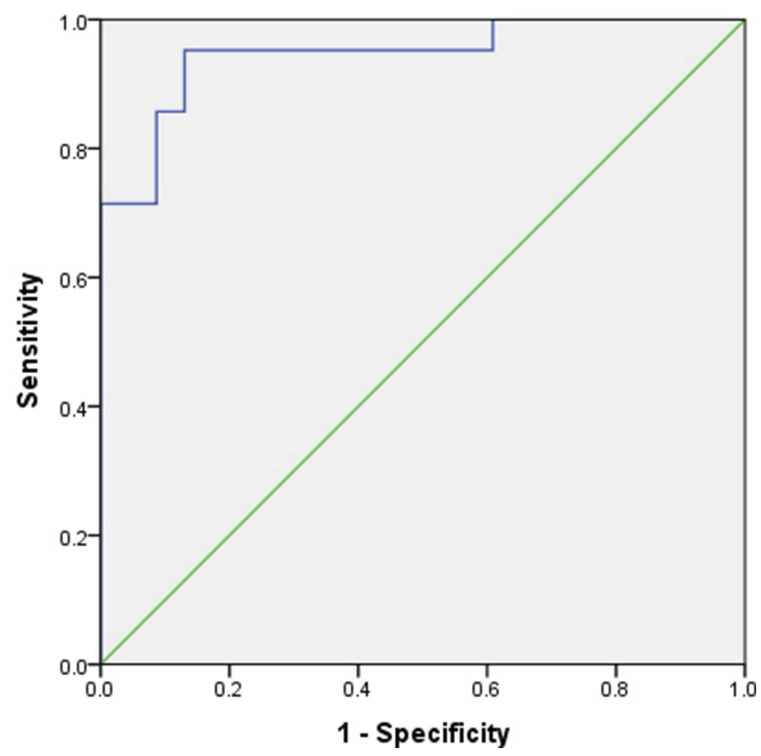

Figure 3 ROC curve analyses correspond to the plasma expression of the two miRNAs to discriminate patients with T-ALL from healthy individuals. (A) The area under the curve of miR-107. (B) The area under the curve of miR-32.

mTOR. Notch1 signaling alteration is strongly related to T-ALL progression. ${ }^{5,24}$ Since malfunction of FBXW7 has been reported in several types of human cancer, this protein seems as an appropriate choice for miRNA targeting studies. Therefore, the focus of the present study was to compare the expression levels of miR-32, miR-107, and FBXW7 using RT-qPCR in the plasma of patients with T-ALL. The miRNAs were chosen using several algorithms that are used for prediction of
mRNA-miRNA interactions. Using DIANA, miRBase, PicTar, Miranda, miRTB, TargetScan, miRDB and miRNApath FBXW7 was predicted to be a target of miR-32, miR-107 and miR-27. However, miR-32 and miR-107 had higher scores and were selected for further experiments.

The plasma levels of miR-32 were significantly higher in the T-ALL group than in the healthy control individuals. However, the plasma level of miR-107 was significantly 
decreased in patients suffering from T-ALL. The expression of miR-32 was upregulated by 5.65 fold $(\mathrm{P}=0.000)$ and expression of miR-107 was downregulated by -2.31 fold $(\mathrm{P}=0.002)$. Furthermore, the plasma levels of FBXW7 were downregulated by 0.013 (or -76.9 fold, $\mathrm{P}=0.000$ ) in the T-ALL group than in the healthy control group. These findings confirm that miR-32 might also boost the proliferation of T-ALL cells by direct-targeting of FBXW7 transcript. On the other hand, the downregulation of miR-107 is inconsistent with the research hypothesis. One of the main plausible explanations might be the fact that bioinformatics tools only predict the intermolecular interactions and their result always need experimental confirmation, as in this case that our data did not support the in silico prediction. Additionally, a few studies have identified mutations in FBXW7 gene which was related to $9-30 \%$ cases of T-cell acute lymphocytic leukemia. ${ }^{7}$ Nevertheless, no mutation is observed in the majority cases of T-ALL and the induction and progression of such cases might be due to overexpression of miR-32 and, hence, suppressing the expression of FBXW7 protein. The bioinformatics predictions presented here indicate that miR-32 could target the transcript of the FBXW7 gene, as the experimental analyses showed the plasma levels of this miRNA were reduced in patients with T-ALL. The role of mir-32 expression has been studied in a few cancer including colorectal cancer, glioma, and nasopharyngeal cancer by targeting PTEN, E2F transcription factor 5, and LATS2, respectively. ${ }^{26-29}$ However, to our knowledge, none of the previous studies evaluated the plasma levels miR-32 as a potential contributing etiology or biomarker of T-ALL. The present study is the first report related to a biological function for this molecule in this type of leukemia.

\section{Conclusion}

The present study showed a negative correlation between upregulation of miR-32 and downregulation of FBXW7 in patients with T-ALL. This might be due to targeting FBXW7 transcript by miR-32. This finding opens the way for a series of future studies in which the reciprocal expression of miR-32 and its target FBXW7 is deeply evaluated and the results may be useful in predicting clinical outcome in T-ALL. Further studies are necessary to thoroughly elucidate the role of the miR-32/FBXW7 interplay, the discovery of their functional relationship might lead to a better understanding of molecular pathways involved in T-ALL, contributing to open new possibilities for future diagnosis, prognosis and therapies in patients affected by acute leukemia. ${ }^{4}$

\section{Acknowledgments}

This study was supported by the Research Deputy of Arak University of Medical Sciences. The authors thank Neda Molaee and Dr. Ali Ganji for their assistance in this research.

\section{Disclosure}

The authors report no conflicts of interest in this work.

\section{References}

1. Mets E, Van Peer G, Van der Meulen J, et al. MicroRNA-128-3p is a novel oncomiR targeting PHF6 in T-cell acute lymphoblastic leukemia. Haematologica. 2014;99(8):1326-1333. doi:10.3324/haematol. 2013.099515

2. Qian L, Zhang W, Lei B, et al. MicroRNA-101 regulates T-cell acute lymphoblastic leukemia progression and chemotherapeutic sensitivity by targeting Notch1. Oncol Rep. 2016;36(5):2511-2516. doi:10.3892/or.20 16.5117

3. Drobna M, Szarzynska-Zawadzka B, Dawidowska M. T-cell acute lymphoblastic leukemia from miRNA perspective: basic concepts, experimental approaches, and potential biomarkers. Blood Rev. 2018;32(6):457-472. doi:10.1016/j.blre.2018.04.003

4. Verduci L, Azzalin G, Gioiosa S, et al. microRNA-181a enhances cell proliferation in acute lymphoblastic leukemia by targeting EGR1. Leuk Res. 2015;39(4):479-485. doi:10.1016/j.leukres.2015.01.010

5. Yeh $\mathrm{CH}$, Bellon M, Nicot C. FBXW7: a critical tumor suppressor of human cancers. Mol Cancer. 2018;17(1):115. doi:10.1186/s12943-018$0857-2$

6. Malyukova A, Brown S, Papa R, et al. FBXW7 regulates glucocorticoid response in T-cell acute lymphoblastic leukaemia by targeting the glucocorticoid receptor for degradation. Leukemia. 2013;27 (5):1053-1062. doi:10.1038/leu.2012.361

7. Van Vlierberghe P, Ferrando A. The molecular basis of $\mathrm{T}$ cell acute lymphoblastic leukemia. J Clin Invest. 2012;122(10):3398-3406. doi:10.1172/JCI61269

8. Lai EC. Two decades of miRNA biology: lessons and challenges. $R N A$ (New York, NY). 2015;21(4):675-677. doi:10.1261/rna.051193.115

9. O'Brien J, Hayder H, Zayed Y, Peng C. Overview of microRNA biogenesis, mechanisms of actions, and circulation. Front Endocrinol (Lausanne). 2018;9:402. doi:10.3389/fendo.2018.00402

10. Wahid F, Shehzad A, Khan T, Kim YY. MicroRNAs: synthesis, mechanism, function, and recent clinical trials. Biochim Biophys Acta. 2010;1803(11):1231-1243. doi:10.1016/j.bbamcr.2010.06.013

11. Lee YS, Dutta A. MicroRNAs in cancer. Annu Rev Pathol. 2009;4:199-227. doi:10.1146/annurev.pathol.4.110807.092222

12. Filipowicz W, Bhattacharyya SN, Sonenberg N. Mechanisms of posttranscriptional regulation by microRNAs: are the answers in sight? Nat Rev Genet. 2008;9(2):102-114. doi:10.1038/nrg2290

13. Rotkrua P, Shimada S, Mogushi K, Akiyama Y, Tanaka H, Yuasa Y. Circulating microRNAs as biomarkers for early detection of diffusetype gastric cancer using a mouse model. Br J Cancer. 2013;108 (4):932-940. doi:10.1038/bjc.2013.30

14. Wallaert A, Van Loocke W, Hernandez L, Taghon T, Speleman F, Van Vlierberghe P. Comprehensive miRNA expression profiling in human T-cell acute lymphoblastic leukemia by small RNA-sequencing. Sci Rep. 2017;7(1):7901. doi:10.1038/s41598-017-08148-x 
15. Ye F. MicroRNA expression and activity in T-cell acute lymphoblastic leukemia. Oncotarget. 2018;9(4):5445-5458. doi:10.18632/oncotarget. v9i4

16. Bene MC, Castoldi G, Knapp W, et al. Proposals for the immunological classification of acute leukemias. European Group for the Immunological Characterization of Leukemias (EGIL). Leukemia. 1995;9(10):1783-1786.

17. Peltier HJ, Latham GJ. Normalization of microRNA expression levels in quantitative RT-PCR assays: identification of suitable reference RNA targets in normal and cancerous human solid tissues. $R N A$ (New York, NY). 2008;14(5):844-852. doi:10.1261/rna.939908

18. Serafin A, Foco L, Blankenburg H, et al. Identification of a set of endogenous reference genes for miRNA expression studies in Parkinson's disease blood samples. BMC Res Notes. 2014;7:715. doi:10.1186/1756-0500-7-715

19. Gharbi S, Shamsara M, Khateri S, et al. Identification of reliable reference genes for quantification of microRNAs in serum samples of sulfur mustard-exposed veterans. Cell J. 2015;17(3):494-501. doi:10. 22074/cellj.2015.9

20. Pfaffl MW. Relative expression software tool (REST) for group-wise comparison and statistical analysis of relative expression results in realtime PCR. Nucleic Acids Res. 2002;30(9):e36. doi:10.1093/nar/30.9.e36

21. Zhang B, Pan X, Cobb GP, Anderson TA. microRNAs as oncogenes and tumor suppressors. Dev Biol. 2007;302(1):1-12. doi:10.1016/j. ydbio.2006.08.028

22. Niloofar Moradi MP, Khansarinejad B, Sarmadian H, Mondanizadeh M. Plasma level of miR-5193 as a novel biomarker for diagnosis of HBVrelated hepatocellular carcinoma. Hepat Mon. 2019;19(2):e84455.
23. Parvaee P, Sarmadian H, Khansarinejad B, Amini M, Mondanizadeh M. Plasma level of microRNAs, miR-107, miR-194 and miR-210 as potential biomarkers for diagnosis intestinal-type gastric cancer in human. Asian Pac J Cancer Prev. 2019;20(5):1421-1426. doi:10.31 557/APJCP.2019.20.5.1421

24. Wallaert A, Durinck K, Taghon T, Van Vlierberghe P, Speleman F. T-ALL and thymocytes: a message of noncoding RNAs. J Hematol Oncol. 2017;10(1):66. doi:10.1186/s13045-017-0432-0

25. Yin JJ, Liang B, Zhan XR. MicroRNA-204 inhibits cell proliferation in T-cell acute lymphoblastic leukemia by down-regulating SOX4. Int J Clin Exp Pathol. 2015;8(8):9189-9195.

26. Jin Y, Cheng H, Cao J, Shen W. MicroRNA 32 promotes cell proliferation, migration, and suppresses apoptosis in colon cancer cells by targeting OTU domain containing 3. J Cell Biochem. 2019;120 (11):18629-18639. doi:10.1002/jcb.v120.11

27. Wang TT, Chen ZZ, Xie P, et al. Isoliquiritigenin suppresses the proliferation and induced apoptosis via miR-32/LATS2/Wnt in nasopharyngeal carcinoma. Eur J Pharmacol. 2019;856:172352. doi:10.1016/j.ejphar.2019.04.033

28. Wu W, Tan W, Ye S, Zhou Y, Quan J. Analysis of the promoter region of the human miR-32 gene in colorectal cancer. Oncol Lett. 2019;17(4):3743-3750. doi:10.3892/ol.2019.10042

29. Zhang Y, Wang J, An W, et al. MiR-32 inhibits proliferation and metastasis by targeting EZH2 in glioma. Technol Cancer Res Treat. 2019;18:1533033819854132. doi:10.1177/15330338198 54132

\section{Publish your work in this journal}

Cancer Management and Research is an international, peer-reviewed open access journal focusing on cancer research and the optimal use of preventative and integrated treatment interventions to achieve improved outcomes, enhanced survival and quality of life for the cancer patient.
The manuscript management system is completely online and includes a very quick and fair peer-review system, which is all easy to use. Visit http://www.dovepress.com/testimonials.php to read real quotes from published authors. 